\title{
Sterile at reactors: PROSPECT
}

\author{
Karin Gilje* for the PROSPECT Collaboration \\ Illinois Institute of Technology \\ E-mail: kgilje@it. edu
}

PROSPECT, the Precision Reactor Oscillation and Spectrum Experiment, is a phased experiment at the High Flux Isotope Reactor of the Oak Ridge National Laboratory. The two primary goals of the experiment are to measure the ${ }^{235} \mathrm{U}$ antineutrino spectrum and to perform a search for sterile neutrinos with a $\Delta m_{14}^{2}$ on the $1 \mathrm{eV}^{2}$ scale. The Phase I detector will cover a baseline range of 7-12 $\mathrm{m}$ from the core while the second phase will extend the baseline out to $20 \mathrm{~m}$. The detection medium for the first phase is 3 -tons of ${ }^{6} \mathrm{Li}$ loaded liquid scintillator in an optically segmented detector. This talk describes the current status of the experiment and the projected sensitivity in the search for an $\mathrm{eV}$-scale sterile neutrino.

Neutrino Oscillation Workshop

4 - 11 September, 2016

Otranto (Lecce, Italy)

\footnotetext{
* Speaker.
} 


\section{Motivation}

Reactor antineutrinos have historically galvanized the field of neutrino physics from the discovery of antineutrino to the precise measurement of the neutrino oscillation mixing angle, $\theta_{13}$. Two methods are used to model the reactor antineutrino spectrum [1,2]. The ab-initio method relies on calculations from nuclear databases to sum the energy of $\bar{v}_{e}$ from all possible decay chains. This method relies on a large databases that are incomplete where some nuclei have large errors on their decay modes and some inconsistencies exist between these databases. The conversion approach uses the measured $\beta$-spectrum to fit to virtual $\beta$ branches to unfold the antineutrino energy. Early measurements suffered from low statistics and large errors. Additionally, it has not been determined if these virtual branches cover all the physics involved in creating the prompt spectra. With reevaluations of the predictions of the reactor antineutrino spectrum in the last decade in conjunction with more precise measurements, two anomalies have appeared. The first is a $\sim 5 \%$ overall antineutrino flux deficit that could be evidence of a sterile neutrino [3]. Indications of a sterile neutrino are not only present in short baseline reactor experiments, but also appear in anomalous behavior in Gallium experiments and long baseline oscillation experiments such as LSND and MiniBooNE. The second is an excess in events in the prompt energy spectrum around $5 \mathrm{MeV}$ seen in Daya Bay, RENO, and Double CHOOZ [4]. New high precision data of reactor antineutrino spectra are needed in order to resolve these issues.

\section{The PROSPECT Experiment}

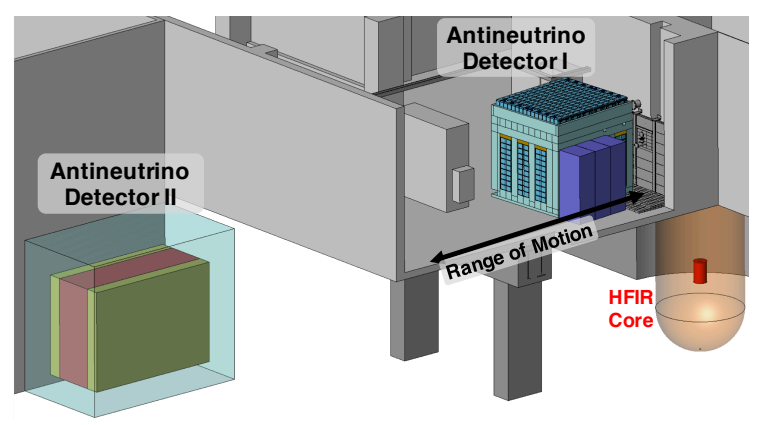

Figure 1: The arrangement of the detectors around the HFIR facility [5].

PROSPECT, the Precision Reactor Oscillation and Spectrum measurement, seeks to resolve both the flux deficit and spectrum shape anomalies. The Phase I detector will be located $\sim 7$ meters from the High Flux Isotope Reactor (HFIR) core at Oak Ridge National Laboratory (ORNL) in Oak Ridge, TN seen in Figure 1. Additionally, the Phase I detector can be moved to cover up to $\sim 12$ meters from the reactor core in order to extend the baseline used to search for sterile neutrinos. HFIR, which operates at $85 \mathrm{MW}$, is ideally suited as a source of antineutrinos due to its compact ( 0.4 meter diameter and 0.5 meter height) size and due to its fuel content. Unlike commercial reactors, which contain a mixture of ${ }^{235} \mathrm{U},{ }^{238} \mathrm{U},{ }^{239} \mathrm{Pu}$, and ${ }^{241} \mathrm{Pu}$, this research reactor is predominantly ${ }^{235} \mathrm{U}$. This has the added benefit of removing any unfolding of the individual fission elements from the spectrum calculation and provides a constant fission fraction through 
the duration of the reactor on cycle. HFIR also has a significant amount of reactor off time $(\sim$ 54\%) which allows for in-depth background studies. Over the last few years, several prototype deployments were performed at HFIR in order to study backgrounds and to test the mechanical components of the detector. The final PROSPECT design was developed in cooperation with HFIR engineers and is presently under construction with first data expected in 2017.

The Phase I detector will be an array of 120 segments that are $14.6 \times 14.6 \times 119 \mathrm{~cm}$. Each end of the segment will have a $12.7 \mathrm{~cm}$ photomultiplier tube. This double ended readout improves both the energy and position reconstruction. The segments are optically separated by reflective panels held in place by pinwheel supports. The corner supports also provide locations for source deployments in order to calibrate the detector in situ. The detector medium has a commercial liquid scintillator base, EJ-309, that has been doped with ${ }^{6} \mathrm{Li}$ as a neutron target. In the event of an upgrade, a Phase II detector will be constructed using the same base segment but four times the size. It would be placed outside the HFIR building in order to extend the baseline from the reactor up to 20 meters.

Due to the location of the detector on the surface, the dominant backgrounds come from cosmogonics and from the reactor. To reduce these backgrounds, a multi-layered shield has been developed of water, lead and polyethylene. The segmentation provides additional rejection of background events through the use of a veto layer on the outer edge of the detector. The fiducial volume will have $\sim 1500 \mathrm{~kg}$ of target mass. The event signature of interest comes from the inverse beta decay (IBD) interaction where $\bar{v}_{e}+p \rightarrow n+e^{+}$. The positron annihilates producing light with energy in the 1 to $10 \mathrm{MeV}$ range. The neutron provides a delay signal at $0.6 \mathrm{MeV}$ electron equivalent energy after capturing on ${ }^{6} \mathrm{Li}$. Using the temporal and spatial coincidences, the final efficiency of the detection is $42 \%$ with a signal to background ratio of 3 to 1 . The Phase I detector expects to record 115,000 IBD events in the first year of data taking. The predicted energy resolution is $4.5 \% / \sqrt{E}$. Further details of the projected detector performance can be found in [5].

\section{Oscillation Sensitivity}

The sensitivity of the PROSPECT experiment can be seen in Figure 2. By comparing the $3+$ $1 v$ oscillation model $\left(M_{i j}\right)$ to the standard $3 v$ oscillation model $\left(T_{i j}\right)$ with an irreducible background $\left(B_{i j}\right)$, a $\chi^{2}$ minimization was performed with

$$
\chi^{2}=\sum_{i, j} \frac{\left(M_{i j}-\left(\alpha+\alpha_{e}^{i}+\alpha_{r}^{j}\right) T_{i j}+\left(1+\alpha_{b}\right) B_{i j}\right)^{2}}{T_{i j}+B_{i j}+\sigma_{b 2 b}^{2}\left(T_{i j}+B_{i j}\right)^{2}}+\left(\frac{\alpha}{\sigma}\right)^{2}+\sum_{j}\left(\frac{\alpha_{r}^{j}}{\sigma_{r}}\right)^{2}+\sum_{i}\left(\frac{\alpha_{e}^{i}}{\sigma_{e}}\right)^{2}+\left(\frac{\alpha_{b}}{\sigma_{b}}\right)^{2}
$$

where parameters $\alpha$ account for systematic uncertainties in the signal and the background. Specifically,

$$
\left(\sigma, \sigma_{b}, \sigma_{e}, \sigma_{r}, \sigma_{b 2 b}\right)=(100 \%, 2 \%, 10 \%, 1 \%, 1 \%)
$$

for the reactor flux normalization, the background normalization, the reactor spectrum shape, a position-dependent variation and a bin-to-bin correction respectively. Exclusion contours were calculated in the $\left(\Delta m_{41}^{2}, \theta_{14}\right)$ parameter space as seen in Figure 2. Within one year of PROSPECT 


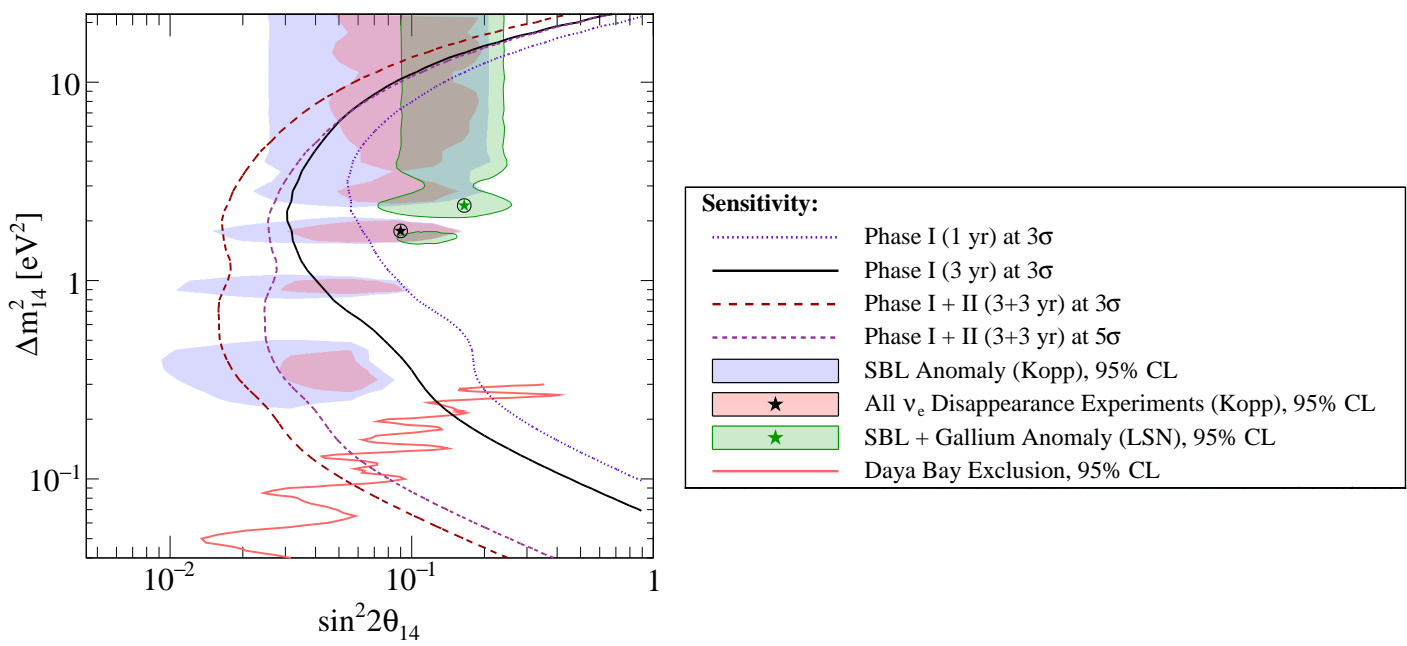

Figure 2: The predicted sensitivity reach into the $\Delta m_{14}^{2}-\sin ^{2} 2 \theta_{14}$ parameter space. Shown are the $3 \sigma$ sensitivity curves for Phase I at 1 and 3 years of data taking and the $3 \sigma$ and $5 \sigma$ curves for Phase II with an additional 3 years of data taking [5]. The Daya Bay contour is from [6].

data taking, the best fit will have $3 \sigma$ coverage. Within six years of Phase I + II, the majority of the current anomalous region around $\delta m_{14}^{2} \sim 1 \mathrm{eV}^{2}$ will have at least $3 \sigma$ coverage.

\section{Acknowledgments}

This material is based upon work supported by the U.S. Department of Energy Office of Science. Additional support for this work is provided by Yale University, the Illinois Institute of Technology, the National Institute of Standards and Technology, and the Lawrence Livermore National Laboratory LDRD program. We gratefully acknowledge the support and hospitality of the High Flux Isotope Reactor at Oak Ridge National Laboratory, managed by UT-Battelle for the U.S. Department of Energy.

\section{References}

[1] T. Mueller et al., Improved predictions of reactor antineutrino spectra, Phys. Rev. C 83 (5) 054615 [hep-ex/1101.2663v3]

[2] P. Huber, On the determination of anti-neutrino spectra from nuclear reactors, Phys. Rev. C 84 (2) 024617 [hep-ph/1106.0687v4]

[3] J. Kopp et al., Sterile neutrino oscillations: the global picture, J. High Energy Phys. 2013 (5) 50 [hep-ph/1303.3011]

[4] D. Dwyer and T. Langford, Spectral structure of electron antineutrinos from nuclear reactors, Phys. Rev. Lett. 114 (1) 012502 [nucl-ex/1407.1281]

[5] PROSPECT Collaboration, The PROSPECT physics program, J. Phys. G 43 (11) 113001 [physics.ins-det/1512.02202v1]

[6] Daya Bay Collaboration, Search for a light sterile neutrino at Daya Bay, Phys. Rev. Lett. 113 (14) $14802[$ hep-ex/1407.7259] 